\title{
A bedside feasibility study with optical coherence tomography for real-time tumor-located of lung cancer
}

\author{
Miao-Hui Lin ${ }^{1}$, Hung-Chang Liu ${ }^{2}$, Tien-Yu Hsiao ${ }^{1}$, Ching-Heng Ting ${ }^{3}$, Chia-Wei Sun ${ }^{1}$ \\ ${ }^{1}$ Biomedical Optical Imaging Lab, Department of Photonics and Institute of Electro-Optical Engineering, National Chiao Tung University, Hsinchu \\ City, Taiwan; ${ }^{2}$ Section of Thoracic Surgery, Mackay Memorial Hospital, Taipei City, Taiwan; ${ }^{3}$ Department of Pathology, Mackay Memorial Hospital, \\ Taipei City, Taiwan \\ Contributions: (I) Conception and design: All authors; (II) Administrative support: HC Liu, CW Sun; (III) Provision of study material or patients: MH \\ Lin, TY Hsiao; (IV) Collection and assembly of data: MH Lin, HC Liu; (V) Data analysis and interpretation: MH Lin, CH Ting; (VI) Manuscript \\ writing: All authors; (VII) Final approval of manuscript: All authors \\ Correspondence to: Chia-Wei Sun; Hung-Chang Liu. Biomedical Optical Imaging Lab, Department of Photonics and Institute of Electro-Optical \\ Engineering, National Chiao Tung University, No. 1001, University Rd, East District, Hsinchu City, 300, Taiwan. \\ Email: chiaweisun@nctu.edu.tw; oncoteam@yahoo.com.
}

Background: Instant histological diagnosis is an important procedure during surgery for histologyunknown lung tumors. Through such a brief diagnosis, accurate decision-making excision of the target, especially in lung cancer, is crucial for the cure of the disease. There is still a limited advancement in this kind of microscopic diagnostic technology during these years.

Methods: We design a spectral domain optical coherence tomography (SD-OCT) system and perform the real-time scanning of targeting tumor intraoperatively. The images got from this novel system are compared with the results by the frozen section and permanent pathological process. Characteristics and weaknesses of OCT-captured images for lung tumors are analyzed and presented.

Results: A total of 6 ex vivo lung specimens from 3 patients, diagnosed with invasive adenocarcinoma (IA), were enrolled in this study. Depending on appropriate signal processing methods, the normal and cancerous regions showed different features of OCT images. This proposed optical method can provide an instant micrometer level scope of tissue images. By comparing OCT images of the cross-section and enface, intensity clefs and dark stripes can be observed below the surface of the cancerous part. We inferred that these are very classic features of tumors. The corresponding regions can be identified on OCT-scanned images as well as those from the H\&E stain results.

Conclusions: From the results of this study, an optical imaging method by building an instantly on-site support through this novel modality was possible and feasible. Furthermore, we expect that future deep learning ability of OCT-captured images could be carried out to avoid time-consuming and resourceconsuming status and improve current diagnostic efficiency.

Keywords: Lung cancer; optical coherence tomography (OCT); tumor demarcation

Received: 20 February 2020; Accepted: 17 November 2020; Published: 30 April 2021.

doi: 10.21037/ht-20-10

View this article at: http://dx.doi.org/10.21037/ht-20-10

\section{Introduction}

Lung cancer is the first leading cause of cancer-related mortality in the United States, with over 220 thousand new cases diagnosed and over 140 thousand deaths projected in 2019 (1). The earlier diagnosis of the disease will result in a higher possibility of the cure. Surgery is thought to be the best treatment option for early lung cancers (2). with the advance of diagnostic image tools, more early and small tumors can be found during a regular health check. Therefore, the role of instant intraoperative diagnosis is more 


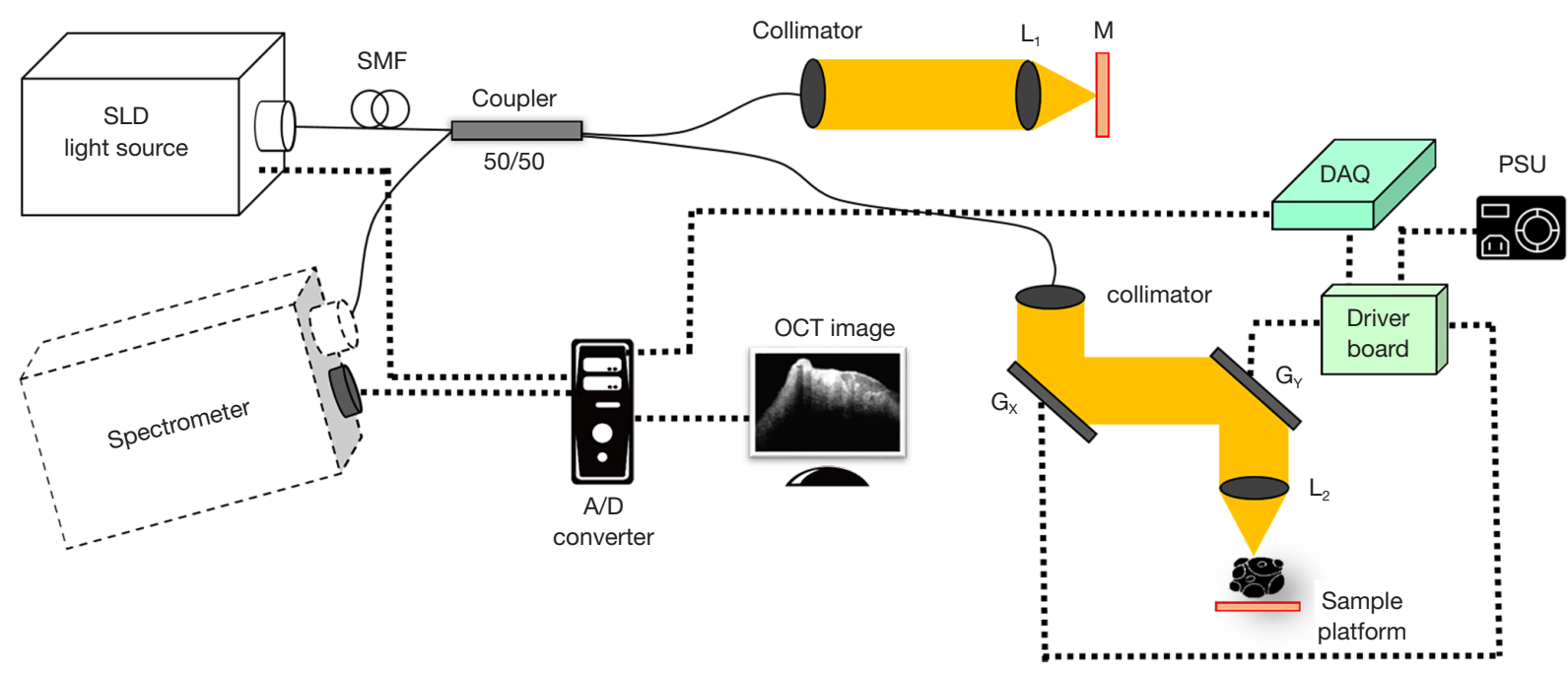

Figure 1 Schematic diagram of the SD-OCT. SMF, single mode fiber; $L_{1}, L_{2}$, achromat lens; $M_{1}$, mirror; $G_{X}, G_{Y}$, galvo-scanners; DAQ, data acquisition (NI-6343, National Instruments); PSU, power supply.

and more important. However, microscopic resection-ensured and margin-free identification of these early and small tumors becomes a relatively tough issue because the procedure mainly depends on integrated information from preoperative imaging study plus direct visualization and palpation. Pathological techniques to examine the margins intraoperatively, such as frozen section analysis (FSA) (3) and imprint cytology, have been widely used to reduce the conditions of positive margins. However, all these methods have certain drawbacks, such as time-consuming or human resource-required process (4), and sometimes even false adequate resection, due to surgical sampling errors. All these unwanted limitations may induce beyond tumor resection, microscopic residual tumors and increased risks of local tumor recurrence (5).

Optical coherence tomography (OCT) was photometric technology ever developed for harmless cross-sectional imaging of biological systems (6). OCT is non-contact, noninvasive, and non-radiative, providing real-time micrometer imaging without the need for special preparation. It was first introduced in 1991 by Huang et al. (7). This imaging technique base on the principle of low coherence interferometry, and the contrast mechanism of OCT relies on the variations of refractive index and the light scattering properties associated with different tissue structures. By detection and transformation of the data from the backscattering light beam over the tissue, twodimensional images or three-dimensional volumetric images can be recorded and analyzed. The utility of this imaging modality was previously demonstrated in ophthalmology (8) and cardiology (9). This kind of optical imaging method offers near histologic resolution for visualizing tissue structures on and below the surface down to the scope of $2-3 \mathrm{~mm}$ (10). The resolution lies in the range of $1-20 \mu \mathrm{m}$ in the axial and transverse direction, depending on the modality used. Although OCT has been used as an optical biopsy tool in certain cancerous areas, such as skin (11) and breast (12), there are limited reports with OCT used in nude specimens of the lung, either malignant or benign. In this study, we tried to explore the potential of OCT and differentiate the fresh lung specimen, especially invasive adenocarcinoma (IA), intraoperatively. The goal was to determine its feasibility of application in clinical practice.

\section{Methods}

\section{System set-up}

The schematic diagram of the spectral domain OCT (SDOCT), a single-mode fiber-based unbalanced Michelson interferometer, is shown in Figure 1. Light was first emitted from a broadband super luminescent diode (SLD), operating at $840 \mathrm{~nm}$ central wavelength with spectral width (FWHM) $51.2 \mathrm{~nm}$, and $10 \mathrm{~mW}$ max output power. (cBLMD-S-371-HP2-SM-OI, Superlum BroadLighters) The axial resolution was $6.06 \mu \mathrm{m}$ in air. One fiber coupler was assembled. The light from the source entered the 


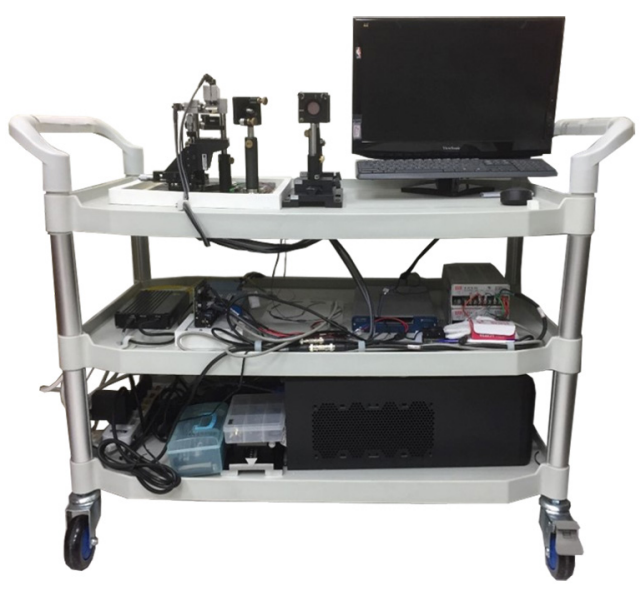

Figure 2 Visible cart picture.

coupler with a splitting ratio of 50:50. In the sample arm, the power of probe beams on the platform was $3 \mathrm{~mW}$. For a $3.99 \mathrm{~mm}$ beam diameter incident on an achromatic lens, the transversal resolution of the spot on the sample was $10 \mu \mathrm{m}$. To facilitate image acquisition, a set of two galvoscanners $\left(G_{X}, G_{Y}\right)$ were installed in the sample arm. (GVS001 and GVS002, THORLABS) With a variable neutral density filter (ND-filter) on the reference path, the power was optimized to maximize the sensitivity of the system. The measured sensitivity was $90 \mathrm{~dB}$. The backscattered probe beam and reflected reference beam were recombined by the coupler and then introduced into the high-speed spectrometer (Cobra-800-880, Wasatch Photonics), composed of a transmission grating and a charge-coupled device (CCD) line scan camera. The line scan camera had 4,096 pixels and could be driven a line rate of up to $40 \mathrm{kHz}$. The data was transferred to a personal computer (PC) via Camera Link connection. The cart visible picture is shown in Figure 2.

In our practical scanning, the speed was $20 \mathrm{k}$ axial scans per second at 2048 pixels. The $G_{X}$ was driven for 2,000 lines, requires $0.1 \mathrm{~s}$. With $G_{Y}$ driven at $0.025 \mathrm{~Hz}, 400$ cross section OCT images were reconstructed at different lateral positions, requires 40 seconds per volume. The volumetric imaging was performed in an area of $2.5 \mathrm{~mm} \times 2.5 \mathrm{~mm}$, with a limited depth of $2.3 \mathrm{~mm}$.

\section{Image acquisition}

Patients being preoperative diagnosis as lung cancer were enrolled in the study. This study was approved by Institutional Review Board for the Protection of Human Subjects of the MacKay Memorial Hospital (18MMHIS084). The trail is not performed in-vivo. Those who have signed the Hospital (Clinic) Surgery Consent Form and sent the specimen to the clinical laboratory shall be deemed to have consented. OCT scanning procedures were conducted intraoperatively. Before operations, the OCT system was shifted the operating room and did fast calibration and optimization of hardware. The surgeon then removed the suspected lesions according to CT images and gross examination. The clinician straightaway gave us fresh specimens after resection. We performed OCT real-time scanning before the frozen section and then data acquisition.

After OCT imaging, specimens were processed and sectioned as the usual standard histology procedures, stained check with hematoxylin-eosin (H\&E). The two kinds of images, OCT-acquired and traditional histologicalprocessed, were analyzed as the endpoints of this study.

\section{Signal processing}

SD-OCT requires the spectral interferometric fringe to be evenly sampled in terms of wavenumber $k$ to reconstruct the axial profile of the tissue. In general, the detected interferometric spectra are resampled (13). A schematic diagram of the processing method is shown in Figure 3. After fast Fourier transform (FFT) and window cropping, sequential A-scan would assemble a preliminary image. However, there still existed some artifacts; for this, we introduced a new algorithm to remove them. Finally, we combined sequential B-scan to a volume set, which could be slice arbitrary in the sagittal section, coronal section, or even horizontal section, usually called en-face. To assist with a large number of data and images, LabVIEW 2019 and ImageJ software was used to facilitate slice stacking, transformation, and 3D volume reconstruction.

\section{Scientific implementation algorithm}

We presented an algorithm to remove artifacts from preliminary OCT images. Figure 4 exemplifies one image processing this algorithm. First, we definite some variables and parameters. $I[m \times n]$ and $I^{*}[m \times n]$ describe the intensity, size $M \times N$, before and after optimization. $m \in\{1, \ldots, M\}$ and $n \in\{1, \ldots, N\}$. $M$ and $N$ are row length and column length of one B-scan. G[m], a binary 1D array of A-scan. X[m×n], a binary 2D array of B-scan. S[n], 1D coordinate composed of 


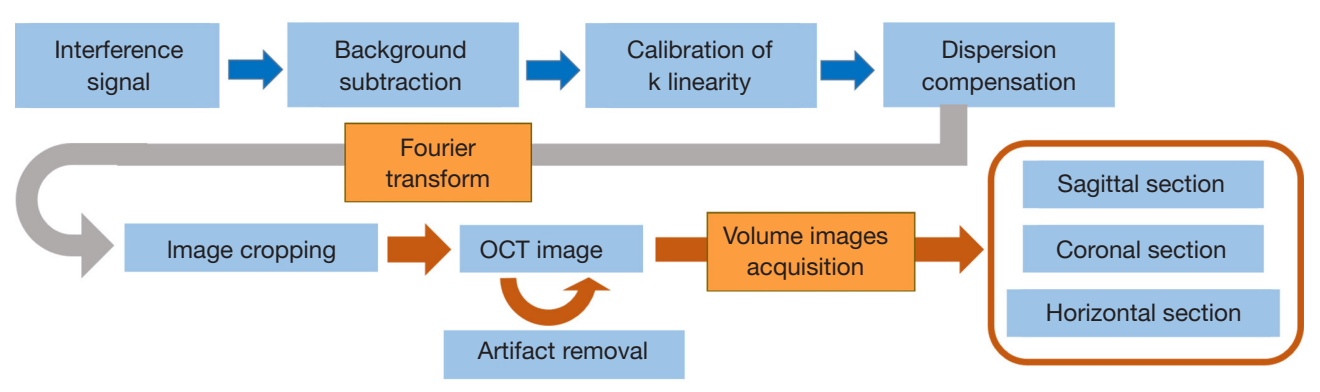

Figure 3 Signal processing procedure.

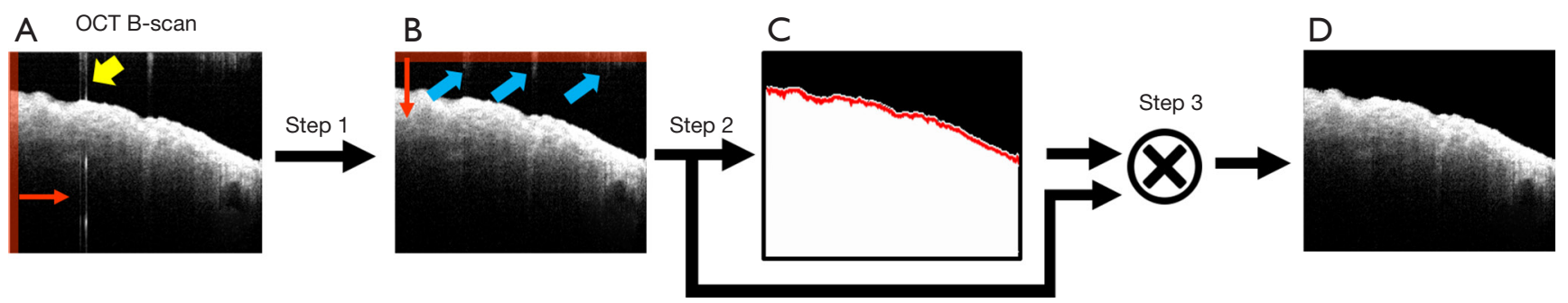

Figure 4 Algorithm interpretation of image optimization. Yellow arrow: strong straight artifact. Blue arrows: residual artifacts. Red arrow: processing direction. Red curve: surface profile.

each A-scan. The bold emphasis denotes that the function is discrete. A detailed description of each step of the algorithm follows.

\section{Step 1: straight artifacts removal}

In general, straight artifacts would exist due to hardware instability and extremely strong reflectivity of tissue top surface, decreasing image quality and missing useful information. Straight artifacts were determined independently for each A-scan. We computed two parameters: $n_{t h}$, two standard error above mean background value, and $l_{t h}$, threshold value that judging whether this A-scan existed straight artifact or not. For the value less than $n_{t h}$, return to zero and assign $\mathrm{G}[\mathrm{m}]=0$; while for the value over $n_{t h}$, remain and assign $G[\mathrm{~m}]=1$. After that, summed up the $G[\mathrm{~m}]$ and check whether this A-scan would be classified in a straight artifact or not. If it's true, replace the current A scan with the previous A-scan. Note that the choice of threshold technique may be sample dependent: for example, the presence of fur reflective water surface may necessitate a more complicated surface extraction method.

\section{Step 2: surface profile detection and a mask creation} Unfortunately, there remained some residual artifacts above the surface probably the autocorrelation terms close to DC signals. At this step, we tried to consider a more comprehensive algorithm for surface profile extraction, $\mathrm{S}[\mathrm{n}]$ and then create a mask for the image, $\mathrm{X}[\mathrm{m} \times \mathrm{n}]$. Providing that the index was above the surface, $X[m \times n]=0$; while the index was below the surface, $X[m \times n]=1$. Firstly, we found the mean of the highest five sequential indexes as the surface coordinate. Secondly, to avoid abrupt isolated coordinated, which did not account for the surface, the profile was monitored and smoothed by repeated iteration: replacement and averaging. For simplicity, the recursion was applied and finally, the surface coordinate would be improved and convergent to an optimized one.

Step 3: multiplication preliminary OCT image and the mask

We multiplied this searching mask and intensity, extracted from step 1 . That's the final optimized image.

\section{Results}

A total of 6 ex vivo lung specimens, presumably IA, from 3 patients over 50 years old were enrolled in this study. Figure $5 A$ showed one the visible picture of a tumor margin right after the excision. Figure $5 B$ demonstrated our two-dimensional (2D) scanning direction, $\mathrm{x}$ and three- 

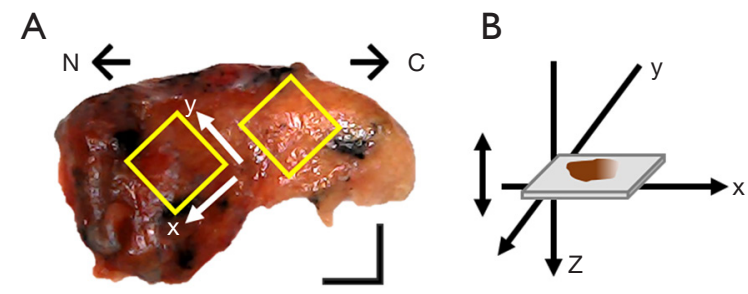

Figure $5 \mathrm{Ex}$ vivo specimen for OCT intraoperative scanning. (A) Visible picture of specimen. N, normal site; C, cancerous site. Yellow boxes: OCT-scanned area, scale bar: $2 \mathrm{~mm}$ and (B) scanning direction demonstration.

dimensional (3D) scanning direction, y. A cross section OCT image was sliced orthogonally to the specimen surface, and 400 cross section images were reconstructed into a complete volume set. To match OCT results, the specimens were sliced at 90 degrees to lesion surface retaining orientation by histologists. OCT sagittal section images of the normal and the cancerous are shown in Figures 6 and 7, including their corresponding H\&E stain images. Via comparing these two kinds of OCT images, the results depicted inhomogeneous to the cancerous side below the surface, while it's homogeneous to the other side.

\section{Image interpretation of $H \& E$-stained vs. OCT-scanned for normal lung tissue}

As the attenuation of near-infrared (NIR) light in biological specimens is governed by Beer-Lambert Law, the attenuation situation is similar at each position owing to well-organized tissue structures. Therefore, the normal region showed homogeneous signal attenuation along with depth. In other words, uniform density tissues would share identical attenuated situation.

\section{Image interpretation of H\&E-stained vs. OCT-scanned for adenocarcinoma of the lung}

The tumor features of the cross section, defined as intensity clefs or dark stripes, can be observed owing to irregular scattering. As going in-depth, repetitive inhomogeneous features different from the surrounding region represent an abnormal reduction of signal strengths.

The OCT-imaged features of necrosis can be identified by lesions that change in texture, contour, and structure. On the other hand, the H\&E stained image showed accumulated and concentrated cellularity with hyper-
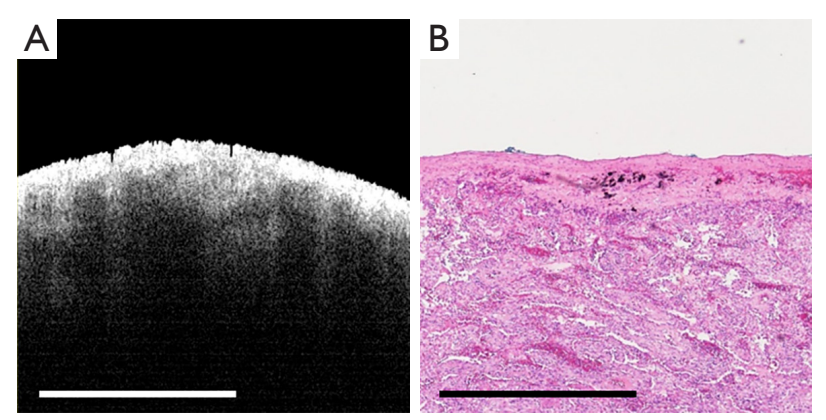

Figure 6 Cross section of the normal region (A) OCT image and (B) $H \& E$ stain image, scale bar: $1 \mathrm{~mm}$.
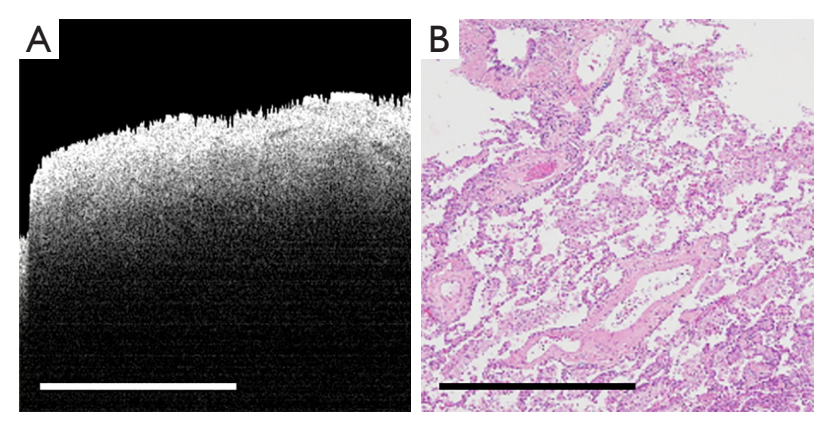

Figure 7 Cross section of the cancerous region (A) OCT image and (B) H\&E stain image, scale bar: $1 \mathrm{~mm}$.

chromosome and disappearance of alveolar air space.

\section{En-face image extraction}

To further allow comparison with OCT and histology, we assembled en-face images, the same displayed direction as general histology. The results of en-face images showed in Figure 8. These OCT images were taken at different depths, measured from the top of the sample in air. From the en-face images, we speculated that noticeable patterns of uneven dark stretches indicated the presence of the tumor.

From Figures 6-8, we inferred that the OCT images are capable of differentiating malignancy and normal from fresh specimens. The normal lung tissue, ordinary cell division, and similar scattering light attenuation were visualized. In contrast, the cancerous region was recognized as heterogeneity due to abnormal hyperplasia.

\section{Discussion}

Lung cancer is globally the most common cause of cancer deaths. During the last decades, many kinds of advanced 

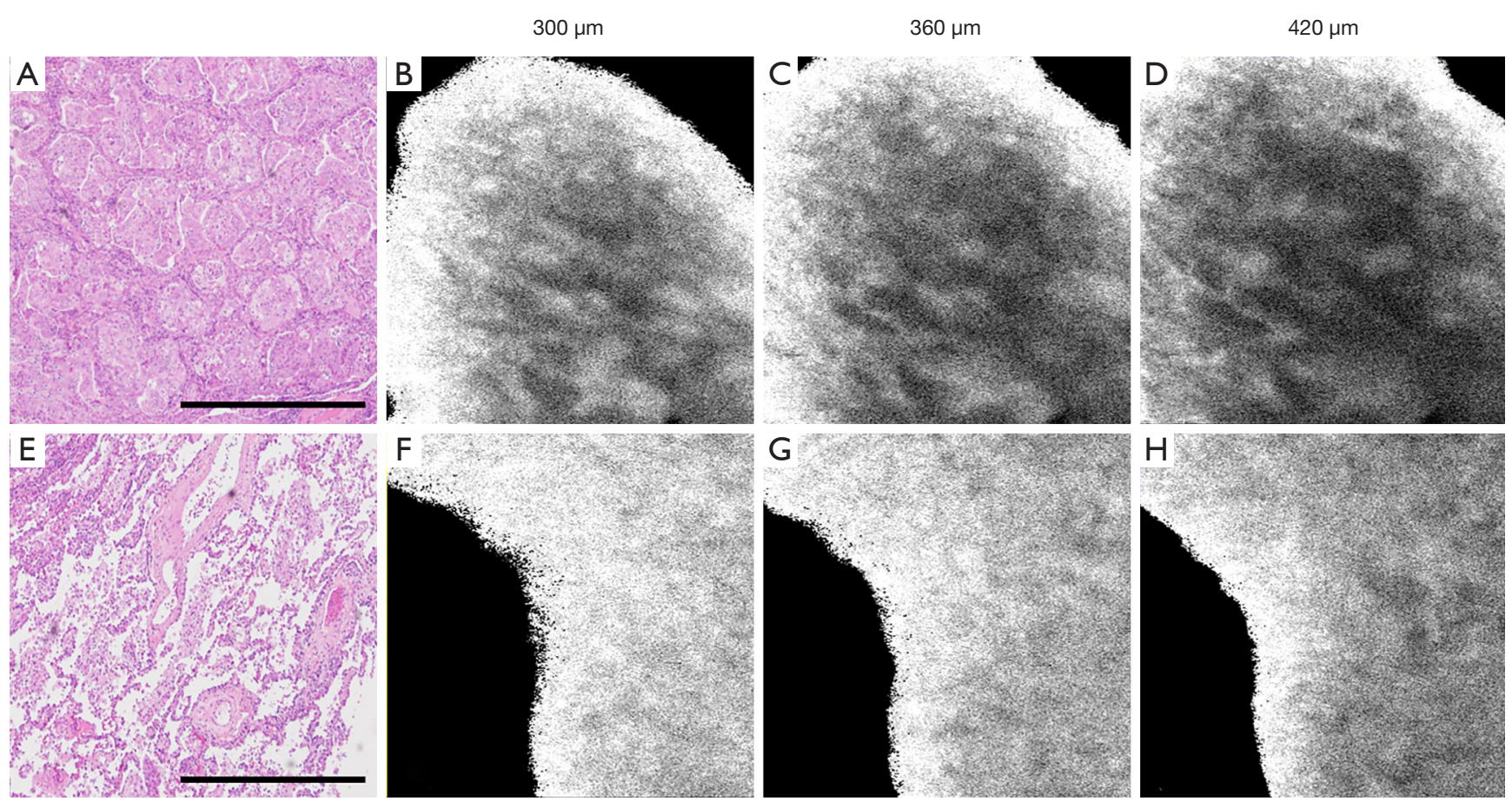

Figure 8 (A,B,C,D) En-face of the cancerous region and (E,F,G,H) en-face of the normal region. (A) and (E) are H\&E stain image. $300 \mu \mathrm{m}$, $360 \mu \mathrm{m}$, and $420 \mu \mathrm{m}$ represent their depth from the top of the specimen, scale bar: $1 \mathrm{~mm}$.

diagnostic imaging tools, such as ultrasound (US), computed tomography (CT), and magnetic resonance imaging (MRI), were built to identify the patient with abnormal lung lesions. However, there remain some disadvantages, for example, low spatial resolution, exposure to ionizing radiation, and high equipment costs. Especially, US examination, which signal disappears due to inappropriate air media in the lung. Also, the number of precancerous early lesions, atypical adenomatous hyperplasia (AAH), and early-stage lung cancer, carcinoma in situ (CIS), is rapidly increased during these years. That means that smart inspection, accurate diagnosis, and adequate treatment play a more important role in the clinical workflow for patients (14). Moreover, pathologic analysis of partial or surrogate tissue from the main tumor target intraoperatively becomes crucial for establishing the near accurate diagnosis and planning subsequent surgical procedures. Except in well facilitated medical center, time-consuming and tissue transportation between operating and laboratory sites are still existent issues clinically. Thus, getting instantly accurate differentiation and on-site diagnosis intraoperatively is imperative.

OCT is an interferometric modality that provides noninvasive tomography of tissues. The first generation of OCT, known as time-domain OCT (TD-OCT), had slow imaging speed, about several hundreds of depth lines per second (15). A recent OCT method, Fourier-domain OCT (FD-OCT) provides faster speed of around several hundred-thousand A-lines per second (16). FD-OCT detects the interference signal in the spectral domain. This spectral domain detection is typically achieved by a highspeed spectrometer or by a wavelength sweeping laser. FDOCT based on the former scheme is called spectral domain OCT (SD-OCT), and that based on the latter scheme is swept-source OCT (SS-OCT). These two kinds of FDOCT have their specific advantages and functions. Owing to SD-OCT's simple schematic diagram and fewer variables, we chose SD-OCT on clinical trials. Furthermore, it's convenient to build the cart system.

In this study, we investigated the feasibility of SDOCT in the real-time evaluation of lung tumors in fresh, unprocessed, and unstained, ex vivo human specimens. We have demonstrated OCT-based technology had the potential to be a pathology assistant. As images from the cross section were inadequate, we further piled up the entire image stack and extracted the transect, en-face images, arbitrarily along with depth. According to our results, OCT- 
captured images of normal tissues showed homogeneous features. In other words, similar light attenuation through lung tissue. In contrast, those of cancerous tissues showed heterogeneity and irregular arrangement. According to the current histologic images, we favored that the reason was abundant tumor cells fill the alveolar space.

Indeed, the lung is a unique organ as it contains lots of blood and air. In the optical window, refers to the wavelength range where the penetration depth of light reaches the maximum in biological tissues, generally in the NIR wavelength range. Within the NIR window, scattering is the most dominant light-tissue interaction. Since scattering increases the distance traveled by photons within the tissue, the probability of photon absorption also increases. The advantage of OCT is that it receives the backscattered light signal. It is worth noting that blood and water absorption have their local minimum in the NIR window. Namely, using NIR as an OCT light source has a relatively smaller effect comparing with other wavelengths. On the other hand, the clinician would use the lung collapse method to turn the hollow organ into a concentrated nearly solid organ, so the specimen may not contain air. Under our OCT measurement, we put the specimen on the suitable objective focal length, $30 \mathrm{~mm}$. It would not be disturbed by air. In our current OCT system, lung sample penetration is about 1.5 to $2 \mathrm{~mm}$.

Additionally, after basic signal processing, calibration of $\mathrm{k}$ linearity (17), FFT, etc. We found that it was significant to do optimization, owing to hardware instability and sample surface moisture, total reflection, artifact, and speckle effect. Hence, we designed a simple algorithm to tweak images and successfully train proper thresholds and parameters. Besides, for any high-resolution imaging setup, there is always an effort to strike a balance between resolution and imaging depth. That is to say, for a high numerical aperture (NA) of the lens would sacrifice depth information. This is non-trivial in OCT since only matched magnification is possible to juxtapose histology microscopes.

This study showed that there was a strong relationship between the surface curve and the power of probe beams hit on the tissue surface. In other words, if the surface curve was located close to the focal plane, with flat and smooth, the power on the sample will be stronger than the surface point away from the focal plane. It was obvious that even if the gap is only one millimeter. For the high-low gap and breach rich sample, it was a more different challenge without flattening the sample. Several studies have shown that using OCT to quantify the local attenuation coefficient was more informative to the pure backscattering intensity.
Yet there were insufficient correct attenuation coefficients in some mediums, especially soft tissues. We have tried some compensated method to regulate the intensity and assess the attenuation coefficient, but the difference between normal and cancerous regions is unsatisfactory.

The clinical studies using OCT for images of the lung are finite. Besides, to differentiate malignant and normal tissues, we expect that OCT can stratify subtypes of malignant lung tumors, even benign inflammation. Furthermore, owing to the lack of clinical human trials, especially the multiinstitution corporation, it is still unable to definitively show the true clinical value of OCT in oncology. To the best of our knowledge, interdisciplinary clinical trial validation could greatly promote the value of the translation of OCT techniques. Since creating a forum where different perspectives can be expressed, good ideas can be put forward, and finally, consensus can be obtained. Moreover, establish the standard operating procedures of OCT methods can build up the atlas of OCT-based oncological imaging. Last but not least, we expect that future learning ability of OCTcaptured images can be carried out to avoid time-consuming and human-resource required situations and able to improve current diagnostic efficiency.

\section{Conclusions}

In summary, this trial was only a pilot study aimed to correlate the histological features of lung cancer and instant OCT-captured images. Based on experiments to characterize malignant histology employing on our novel SD-OCT system, we implemented a besides study. Depending on appropriate signal processing methods, the result warranted a qualitative analysis of normal and cancerous regions from OCT images in the future. We expect that the tumormargin can be demarcated on-site with the OCT technique, instantly, informatively, and efficiently.

\section{Acknowledgments}

Funding: This work was supported by project ' $\mathrm{MMH}$ CT-10808' of interdisciplinary academic research corporation of National Chiao Tung University and Mackay Memorial Hospital, and in part by the Ministry of Science and Technology under Grant Nos. NSC 1062811-E-009-023, NSC 106-2923-E-009-002-MY2, NSC 105-2221-E-009-108-MY3, NSC 105-2811-E-009-028, and NSC 106-2622-E-009-012-CC3. This work was also partially supported by project 'MOST 108-2321-B-010-010- 
MY2' of the Ministry of Science and Technology, Taiwan.

\section{Footnote}

Data Sharing Statement: Available at http://dx.doi. org/10.21037/ht-20-10

Conflicts of Interest: All authors have completed the ICMJE uniform disclosure form (available at http://dx.doi. org/10.21037/ht-20-10). HCL serves as an unpaid editorial board member of Health Technology from Nov 2020 to Oct 2022. The other authors have no conflicts of interest to declare.

Ethical Statement: the authors are accountable for all aspects of the work in ensuring that questions related to the accuracy or integrity of any part of the work are appropriately investigated and resolved. This study was approved by Institutional Review Board for the Protection of Human Subjects of the MacKay Memorial Hospital (18MMHIS084). The trail is not performed in-vivo. Those who have signed the Hospital (Clinic) Surgery Consent Form and sent the specimen to the clinical laboratory shall be deemed to have consented. The trail result is just another image reference indicator for pathological tests.

Open Access Statement: This is an Open Access article distributed in accordance with the Creative Commons Attribution-NonCommercial-NoDerivs 4.0 International License (CC BY-NC-ND 4.0), which permits the noncommercial replication and distribution of the article with the strict proviso that no changes or edits are made and the original work is properly cited (including links to both the formal publication through the relevant DOI and the license). See: https://creativecommons.org/licenses/by-nc-nd/4.0/.

\section{References}

1. Siegel RL, Miller KD, Jemal A. Cancer statistics, 2019. CA Cancer J Clin 2019;69:7-34.

2. Singhal S, Nie S, Wang MD. Nanotechnology applications in surgical oncology. Annu Rev Med 2010;61:359-73.

3. Sienko A, Allen TC, Zander DS, et al. Frozen section of lung specimens. Arch Pathol Lab Med 2005;129:1602-9.

4. Taxy JB. Frozen section and the surgical pathologist: a point of view. Arch Pathol Lab Med 2009;133:1135-8.

5. McLaughlin SA, Ochoa-Frongia LM, Patil SM, et al. Influence of frozen-section analysis of sentinel lymph node and lumpectomy margin status on reoperation rates in patients undergoing breast-conservation therapy. $\mathrm{J}$ Am Coll Surg 2008;206:76-82.

6. Fujimoto JG, De Silvestri S, Ippen EP, et al. Femtosecond optical ranging in biological systems. Opt Lett 1986;11:150.

7. Huang D, Swanson EA, Lin CP, et al. Optical coherence tomography. Science 1991;254:1178-81.

8. Hee MR, Puliafito CA, Wong C, et al. Quantitative assessment of macular edema with optical coherence tomography. Arch Ophthalmol 1995;113:1019-29.

9. Tearney GJ, Brezinski ME, Boppart SA, et al. Images in cardiovascular medicine. Catheter-based optical imaging of a human coronary artery. Circulation 1996;94:3013.

10. Hariri LP, Applegate MB, Mino-Kenudson M, et al. Volumetric optical frequency domain imaging of pulmonary pathology with precise correlation to histopathology. Chest 2013;143:64-74.

11. Nguyen FT, Zysk AM, Chaney EJ, et al. Intraoperative evaluation of breast tumor margins with optical coherence tomography. Cancer Res 2009;69:8790-6.

12. Ferrante di Ruffano L, Dinnes J, Deeks JJ, et al. Optical coherence tomography for diagnosing skin cancer in adults. Cochrane Database Syst Rev 2018;12:CD013189.

13. Yasuno Y, Madjarova VD, Makita S, et al. Threedimensional and high-speed swept-source optical coherence tomography for in vivo investigation of human anterior eye segments. Opt Express 2005;13:10652-64.

14. Orosco RK, Tapia VJ, Califano JA, et al. Positive Surgical Margins in the 10 Most Common Solid Cancers. Sci Rep 2018;8:5686.

15. Rollins A, Yazdanfar S, Kulkarni M, et al. In vivo video rate optical coherence tomography. Opt Express 1998;3:219-29.

16. Potsaid B, Baumann B, Huang D, et al. Ultrahigh speed $1050 \mathrm{~nm}$ swept source/Fourier domain OCT retinal and anterior segment imaging at 100,000 to 400,000 axial scans per second. Opt Express 2010;18:20029-48.

17. Makita S, Fabritius T, Yasuno Y. Full-range, highspeed, high-resolution 1 microm spectral-domain optical coherence tomography using BM-scan for volumetric imaging of the human posterior eye. Opt Express 2008;16:8406-20.

doi: $10.21037 / \mathrm{ht}-20-10$

Cite this article as: Lin MH, Liu HC, Hsiao TY, Ting CH, Sun CW. A bedside feasibility study with optical coherence tomography for real-time tumor-located of lung cancer. Health Technol 2021;5:2. 